\title{
Analyse de l'évolution de l'occupation des terres à partir de photographies aériennes de la localité de Loaga dans la province du Bam, Burkina Faso
}

\author{
Dibi MILLOGO $^{1}$, Abdoul-aziz NIKIEMA ${ }^{1}$, Bazoumana KOULIBALY ${ }^{2 *}$ et \\ Nabsanna Prosper ZOMBRE ${ }^{1}$ \\ ${ }^{1}$ Laboratoire sol, Matériaux et Environnement, UFR/SVT, \\ Université de Ouaga I Pr Joseph Ki-ZERBO, Burkina Faso. \\ 2 Institut de l'Environnement et de Recherches Agricoles (INERA), \\ Programme Coton, 01 BP 208 Bobo-Dioulasso 01, Burkina Faso. \\ *Auteur correspondant ; E-mail : bazoumana@hotmail.com ; Tel : (+226) 70239005.
}

\section{RESUME}

Au Burkina Faso, les contraintes climatiques couplées aux actions anthropiques néfastes ont entraîné une dégradation des ressources naturelles dans la zone du bassin versant du fleuve Nakanbé. L'objectif de l'étude était d'évaluer l'état de dégradation du milieu et d'analyser les effets des mesures entreprises pour y remédier. Des analyses diachroniques de l'occupation des terres en 1982, 1995 et 2008 ont été réalisées par l'interprétation des photographies aériennes de Loaga et ses environs, ainsi que des enquêtes pour appréhender les perceptions paysannes sur les causes, les conséquences et les stratégies d'adaptation aux changements climatiques. Les résultats ont montré que la végétation a régressé de 385 ha de 1982 à 1995 tandis que les terres cultivées ont augmenté durant cette même période. Par contre, de 1995 à 2008, il a été noté un accroissement de la savane claire et une régression des zones cultivées à la suite d'une reprise de la végétation qui a été observée dans les zones aménagées par les techniques de conservation des eaux et des sols (CES). Ces résultats suggèrent de poursuivre les efforts effectués dans la mise en œuvre des techniques CES.

(C) 2017 International Formulae Group. All rights reserved.

Mots clés : Occupation des sols, dégradation, CES, régénération, Burkina Faso.

\section{Analysis of the evolution of soil occupation using aerial photographs at Loaga area in Bam province, Burkina Faso}

\section{ABSTRACT}

In Burkina Faso, climatic constraints in addition to human activities induced the degradation of natural resources, particularly in the catchment of the river Nakanbé. The study objective was to assess the degree of natural resources degradation and to analyze the effects of measures to solve the problem. Diachronic analyses of land occupation in 1982, 1995 and 2008 were carried out through aerial photographs interpretation of Loaga site and surrounding areas. These analyses were coupled with surveys to better understand farmers' perceptions on the causes, consequences and adaptation strategies to climate change. The results showed a decrease of vegetation area of 385 ha from 1982 to 1995, while the area of cultivated lands increased during the same period. In contrast, from 1995 to 2008, an increase in savanna cover and a regression of cultivated lands were 
observed, following land recovery in areas using soil and water conservation (SWC) techniques. These results suggested to continue the ongoing efforts on SWC for environmental restoration.

(C) 2017 International Formulae Group. All rights reserved.

Keywords: Land use, degradation, SWC techniques, vegetation recovery, Burkina Faso.

\section{INTRODUCTION}

Dans la plupart des pays sahéliens d'Afrique sub-saharienne, la majorité de la population, en milieu rural, tire l'essentiel de sa subsistance de l'exploitation des ressources naturelles (Bilgo, 2006; Thiombiano et al., 2012). Le Burkina Faso, pays sahélien, a une économie basée sur la production agropastorale avec $77 \%$ de la population vivant en milieu rural (MAHRH, 2007). L'agriculture et l'élevage emploient $86 \%$ de la population active, fournissent $30 \%$ du produit intérieur brut (PIB) et cumulent $80 \%$ des recettes à l'exportation. Cependant, le pays est soumis depuis plusieurs décennies, à une forte dégradation des ressources naturelles, limitant ainsi le développement des productions agricoles (Belemviré et al., 2008).

Les aspects les plus ressentis par les populations ont été la réduction significative de la couverture végétale, la dégradation des sols, la baisse des rendements agricoles et la diminution des ressources en eau (Zombré, 2006; Lal, 2015; Diallo et al., 2017). A l'étape ultime de la dégradation des terres, il apparaît un sol nu, sur lequel, plus rien ne pousse (Somé et al., 2015).

Le bassin versant du fleuve Nakanbé est une zone de transition entre le sahel et la zone de savane, où l'agriculture et l'élevage occupent une place très importante. Les travaux réalisés par Borrell (2000) dans la zone du bassin versant supérieur du Nakanbé, font apparaître une rapide progression des zones cultivées au détriment de la formation végétale naturelle. La perte du couvert végétal est le plus souvent irréversible et les sols se trouvent ainsi exposés à l'action de l'agressivité climatique, en absence de dispositifs de protection anti-érosive (Conedera et al., 2010; Robert, 2011 ; Zerbo et al., 2017). Par conséquent, il s'y développe des croûtes de battances ou des tâches d'induration, qui favorisent l'érosion et le ruissellement dans cette zone. Ce problème est beaucoup plus crucial dans le Centre-Nord du Burkina Faso, qui, d'après Zombré (2003), est caractérisé par un milieu éco-pédologique contraignant et une pression démographique supérieure à la moyenne nationale. Aussi, le taux annuel élevé de croissance de la population, entraîne de sérieux problèmes de gestion des ressources naturelles et contribue à créer un déséquilibre entre les besoins et la disponibilité en terres cultivables (Zombré, 2006; Yaméogo et al., 2009; Bene et Fournier, 2014). Face à l'insuffisance croissante des terres cultivables, la restauration des terres dégradées et la mise au point des systèmes d'utilisation des terres aptes à préserver les écosystèmes deviennent impérieuses (Somé et al., 2004 ; Da et al., 2008).

Pour résoudre ce problème, les paysans ont développé des initiatives comme les techniques de conservation des eaux et des sols (CES), de défense et restauration des sols (DRS) ou d'agroforesterie (AGF).

La présente étude a pour objectif, d'évaluer, d'une part, les effets des activités agricoles sur l'évolution des ressources naturelles et, d'autre part, d'apprécier l'impact des techniques de lutte endogènes développées dans un terroir de la province du Bam.

\section{MATERIEL ET METHODES}

\section{Présentation du cadre de l'étude}

La présente étude a été conduite à Loaga ( $13^{\circ} 18^{\prime} 53^{\prime \prime} \mathrm{N}$ et $\left.1^{\circ} 37^{\prime} 83^{\prime \prime} \mathrm{W}\right)$, un village de la province du Bam dans la région du Centre-Nord du Burkina Faso (Figure 1). Le climat, de type sahélo-soudanien, est caractérisé par deux saisons bien distinctes: 
une longue saison sèche allant d'octobre à mai et une courte saison pluvieuse allant de juin à septembre.

La province du Bam est constituée de formations cristallines métamorphiques ou volcano sédimentaires, attribuées au précambrien inférieur (Da et al., 2008). Selon la classification française des sols (CPCS, 1967), les sols de cette province appartiennent à cinq classes, à savoir, les sols minéraux bruts, les sols peu évolués, les sols brunifiés, les sols à sesquioxydes de fer et/ou de manganèse et les sols hydromorphes.

Selon Bikienga (2014), la végétation $\mathrm{du}$ Bam appartient au domaine phytogéographique sahélien et se caractérise par une savane arbustive à arborée claire au centre et au sud, qui se densifie au voisinage des axes de drainage. L'aspect et la composition floristique reflètent la résultante du facteur climat, sol et topographie.

\section{Matériel d'étude}

La photographie aérienne de 1982 de la mission IGB 82 037-B Kaya de la ligne $\mathrm{N}^{\circ} 9$ de 2558 à 2559 à l'échelle 1/50 000, celle de 1995 de la mission IGB 95 141-B Kaya de la ligne $\mathrm{N}^{\circ} 21$ de 6650 à 6653 à l'échelle 1/50 000 et une image Landstat extraite à partir de google Earth, ont été les principaux supports utilisés pour les analyses multi-temporelles. Une carte topographique de Kaya à l'échelle 1/50 000, une carte morphopédologique du Centre-Nord à l'échelle 1/50 000 et une carte phytogéographique ont été les supports cartographiques utilisés dans l'étude. En outre, des outils de lecture des photographies aériennes (stéréoscope) et de relevé des coordonnées sur le terrain (GPS GARMIN 12) ont été utilisés.

\section{Enquête sociologique sur les variations climatiques}

Afin de mieux comprendre les manifestations des phénomènes liés aux changements climatiques dans la zone d'étude, une enquête a été réalisée en 2011. Pour ce faire, un questionnaire d'enquête a été administré à un échantillon de 60 personnes vivant sur le terroir de Loaga et ayant au moins 45 ans. Cet échantillon était composé de 50 producteurs ( 40 hommes et 10 femmes), choisis parmi les représentants des comités villageois de gestion des terres, des agropasteurs, des agriculteurs, des éleveurs et des pêcheurs. L'échantillon comportait également les gestionnaires des ressources environnementales, au nombre de 10, composés des chefs de projets de gestion des terres et des techniciens des ministères de l'agriculture, de l'environnement et des ressources animales. Des interviews semistructurées ont été réalisées pour recueillir la perception paysanne des changements climatiques à travers les causes, les manifestations, les conséquences et les stratégies d'adaptation mises en œuvre.

Définition des unités du paysage et interprétation des photographies aériennes

Dans la zone d'étude, en 2011, une description suivie de la définition des unités de paysage homogènes du milieu a été faite à partir des informations disponibles. La délimitation des unités de paysage homogènes a été faite en tenant compte du relief, de l'occupation des terres et de la densité du couvert végétal. Les limites du terroir ont été matérialisées avec l'appui des personnes ressources de Loaga.

L'interprétation des photographies aériennes a consisté en une lecture stéréoscopique et en une analyse déductive des images examinées sur les prises de vue aériennes (PVA) et le dessin des unités identifiées. A l'issue de la photointerprétation, chaque transparent a été assemblé à une photographie aérienne et immobilisé avec du scotch afin de reproduire un plan d'occupation du terroir servant de carte provisoire. Les cartes provisoires et les photographies aériennes ont été utilisées lors des sorties de vérification effectuées sur le terrain afin de corriger les limites des unités préalablement délimitées. Les coordonnées géographiques des points inamovibles tels que les courbures des cours d'eau, les pointes des buttes, les croisements des voies ont été 
relevées à cet effet, à l'aide du GPS GARMIN 12. Ces coordonnées ont été utilisées pour le géo-référencement. Après les vérifications opérées sur le terrain, les cartes provisoires ont été corrigées et numérisées avec le logiciel Map Info 8.5, puis documentées avec Arc View 3.5.

A partir de l'interprétation des photographies aériennes et des travaux de terrain (transects du terroir, visite des sites aménagés en Zaï combiné aux cordons pierreux, les enquêtes avec les notables et les responsables d'organisations paysannes et administratif) et de l'analyse des documents existants, il a été possible de définir des unités de paysage et d'étudier leur évolution en fonction des différentes périodes (1982, 1995 et 2008).

\section{Analyses des données collectées}

Les données collectées lors des enquêtes de terrain ont été saisies, puis analysées à l'aide du logiciel SPSS version 20.0, pour les statistiques descriptives des perceptions des producteurs sur les indicateurs de l'occupation des terres et les changements climatiques. Le traitement des photographies aériennes, à l'effet de produire des cartes, a généré des données numérisées par le logiciel Map Info 8.5.

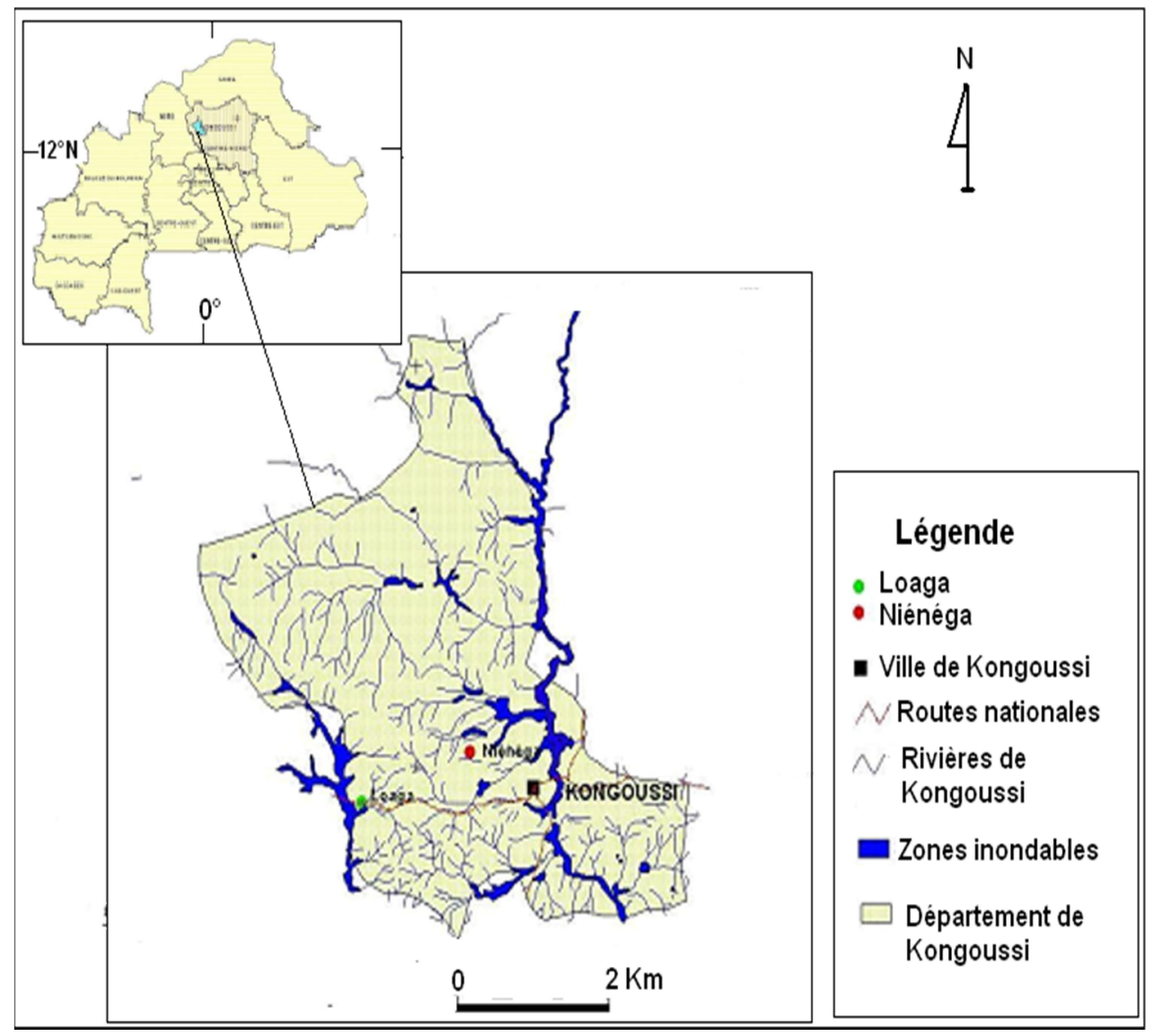

Figure 1: Situation géographique de la zone d'étude (Loaga). 


\section{RÉSULTATS}

Evolution de l'occupation du milieu à partir des photographies aériennes

L'interprétation des photographies aériennes de 1982, 1995 et 2008, a mis en évidence, quatre unités d'occupation des terres (Tableau 1). Il s'agit des zones de cultures, des savanes claires, des buttes et des zones nues. L'examen de ces photographies aériennes n'a pas permis de réaliser une discrimination entre les champs et les jachères. La superficie totale examinée pendant les trois périodes d'observation était de 4764 ha (Tableau 1). Les résultats ont révélé l'absence des savanes boisées dans le terroir de Loaga en 1982, alors que les savanes claires ne recouvraient que 1167,99 ha, soit $24,52 \%$ de la superficie prospectée (Figure 2). En 1995 (Figure 3), il a été noté une réduction de plus de $10 \%$ de la superficie totale de savane claire, estimée à 683,34 ha. Par contre, en 2008 (Figure 4), cette tendance s'est inversée, car la superficie de la savane claire a plutôt connu une hausse de plus de $4 \%$, représentant 887,23 ha.

La superficie des champs de 1982 à 1995 a augmenté de plus $14,62 \%$, en passant de 1857 à 2555 ha. En 2008, les zones mises en culture, estimées à 2351 ha, ont baissé d'environ $4,27 \%$ par rapport aux superficies de 1995. La superficie des buttes représentant des zones inaptes à l'agriculture, est restée inchangée de 1982 à 2008. La superficie des zones nues estimée à 214 ha en 1982, a disparu lors des prospections réalisées de 1995 à 2008, suite aux techniques de conservation des eaux et des sols (CES) pratiquées dans le terroir de Loaga.

\section{Perceptions des manifestations des variations climatiques}

Dans le terroir de Loaga, $82 \%$ des enquêtés ont témoigné que la savane avant les années 1970 était dense et sa limite était à quelques centaines de mètres des concessions (Tableau 2). Cette savane claire regorgeait des espèces fourragères très appétées comme
Pterocarpus spp auxquelles les cheptels avaient accès en quantité et en qualité, sans avoir besoin de se déplacer sur de longues distances. La végétation était si dense qu'elle abritait une faune diversifiée d'herbivores (éléphants, buffles) et de carnivores (lions, hyènes, etc.).

Selon $94 \%$ des enquêtés, après les années 1970, le climat a connu de profonds changements, devenant instable et imprévisible. Les années de sécheresse comme celles observées en 1974, 1978, 1982, 1983, 1984, 1985 et 2011 ont été de plus en plus fréquentes (Tableau 2). Ces vagues de sécheresse ont provoqué une dégradation des ressources naturelles selon $82 \%$ des enquêtés. Les savanes disparaissent à grande échelle, les plantes médicinales, fourragères, celles à fruits comestibles sont menacées de disparition. D'après $66 \%$ des producteurs, cela a occasionné la perte de nombreuses espèces ligneuses utilitaires telles que Vitellaria paradoxa, Adansonia digitata, Bombax costatum, Anogeissus leocarpus, Pterocarpus lucens, et Cassia sieberiana etc. Les espèces herbacées appétées telles que Andropogon gayanus, Andropogon ascinodis ont été également affectées. Les herbacées indicatrices d'une baisse de la fertilité telles que Striga hermonthica, Loudetia togoensis, et Eragrotis tremula sont devenues des espèces les plus fréquemment rencontrées dans les champs selon $66 \%$ des interviewés. Par conséquent, on assiste à une expansion des zones nues après les années 1970 selon $86 \%$ des interrogés, alors que $66 \%$ soulignent qu'il n'y avait pas de zones nues avant cette période. Pour $50 \%$ des personnes interrogées, la végétation s'est reconstituée progressivement dans les champs aménagés avec des diguettes en pierres et en zaï, alors que les zones de pâturages continuaient de se dégrader. Les enquêtés étaient unanimes qu'avec l'application de la technique du zaï combinée aux cordons pierreux, les sols nus se transformaient progressivement en champs générant de bons rendements. 
Tableau 1: Variations des superficies des unités d'occupation du sol du site de Loaga en 1982, 1995 et 2008.

\begin{tabular}{lllllllll}
\hline Unités d'occupation & \multicolumn{2}{c}{$\mathbf{1 9 8 2}$} & & \multicolumn{2}{c}{$\mathbf{1 9 9 5}$} & & \multicolumn{2}{c}{$\mathbf{2 0 0 8}$} \\
\cline { 2 - 3 } du milieu & ha & $\mathbf{( \% )}$ & & ha & $\mathbf{( \% )}$ & & ha & $\mathbf{( \% )}$ \\
\hline Zones des cultures & 1857 & 39 & & 2555 & 53,62 & & 2351 & 49,35 \\
Savanes claires & 1168 & 24,5 & & 684 & 14,38 & & 888 & 18,65 \\
Buttes cuirassées & 1525 & 32 & & 1525 & 32 & & 1525 & 32 \\
Zones nues & 214 & 4,5 & & 0 & 0 & & 0 & 0 \\
\hline
\end{tabular}

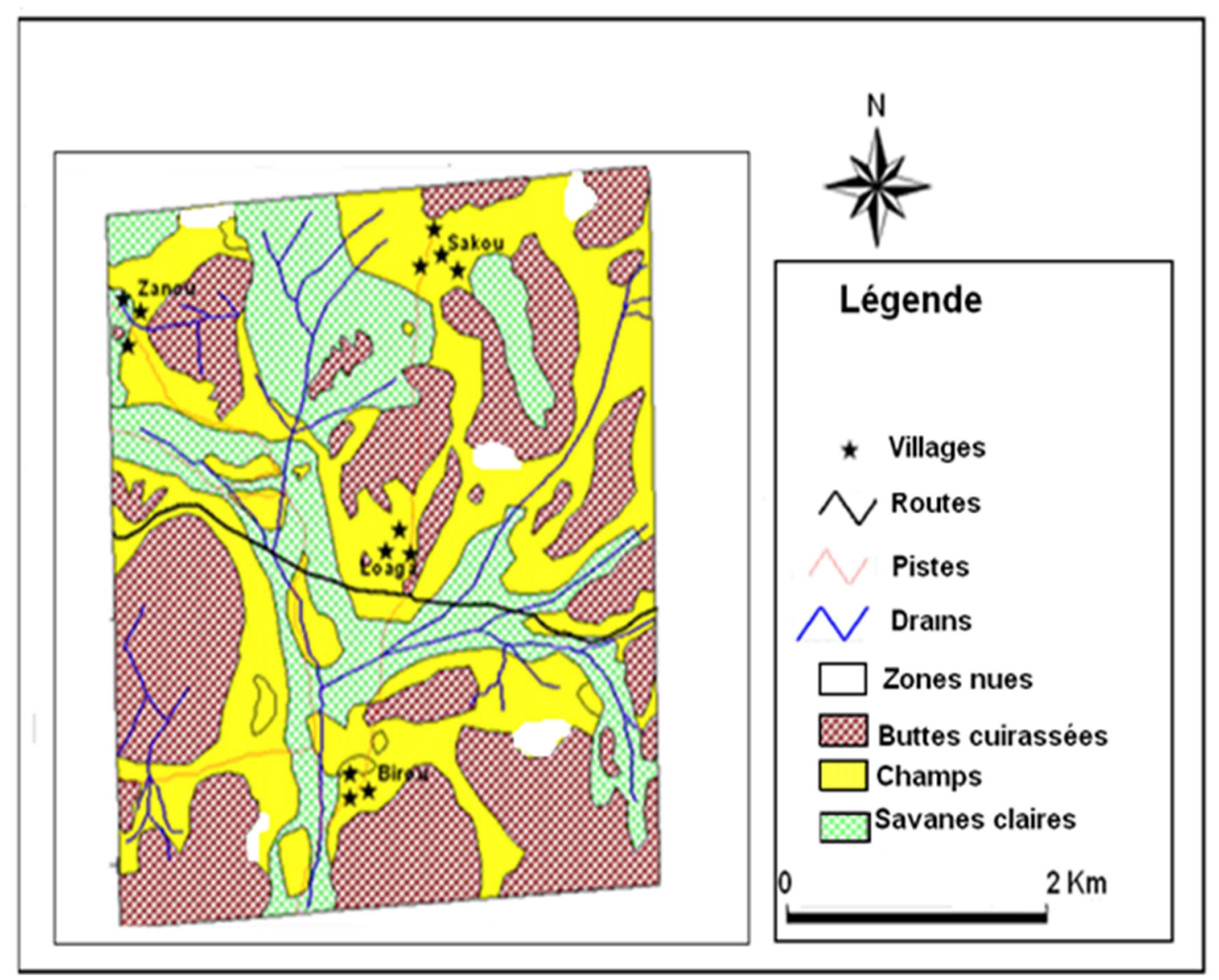

Figure 2: Carte d'occupation des terres de Loaga en 1982. 


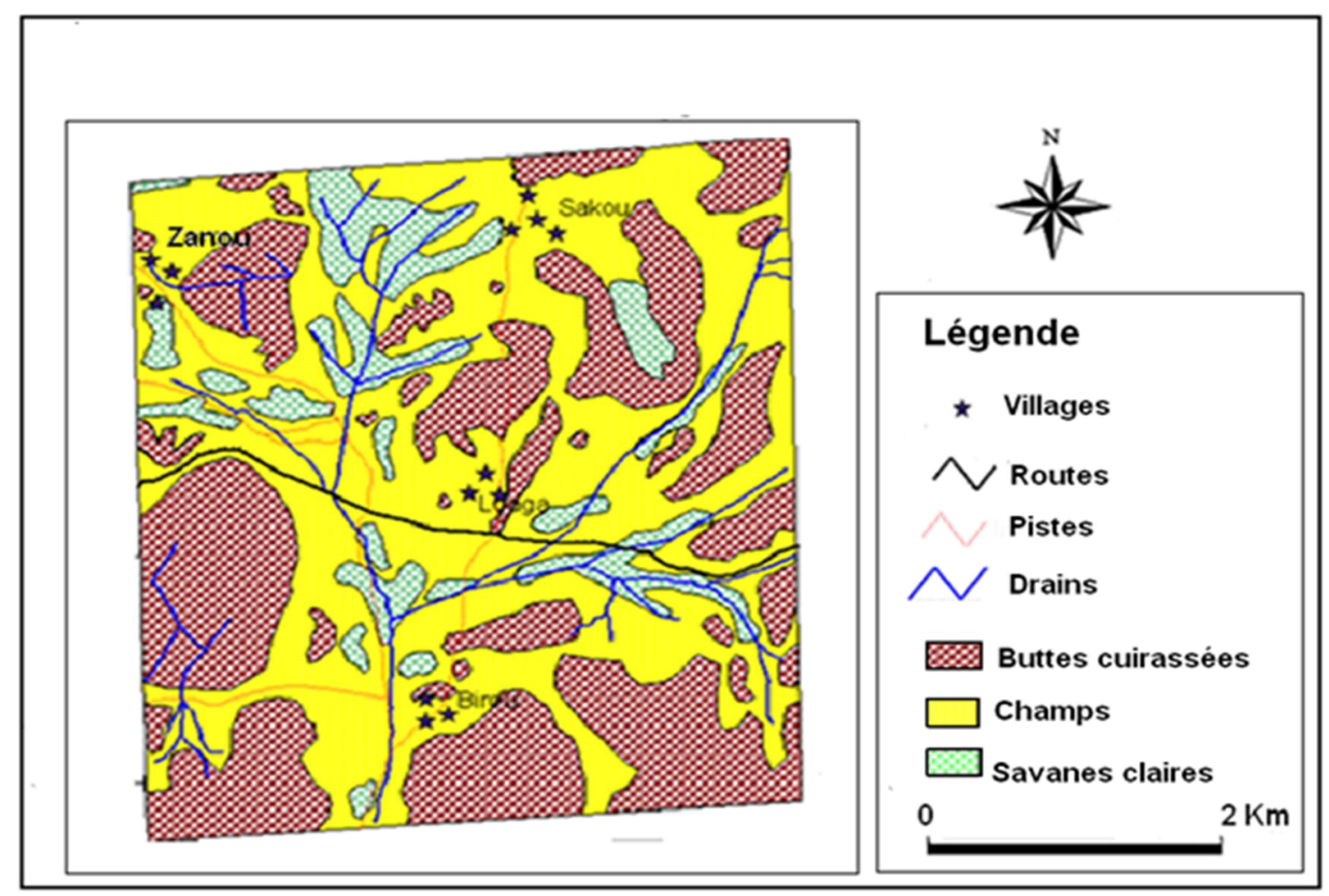

Figure 3: Carte d'occupation des terres de Loaga en 1995.

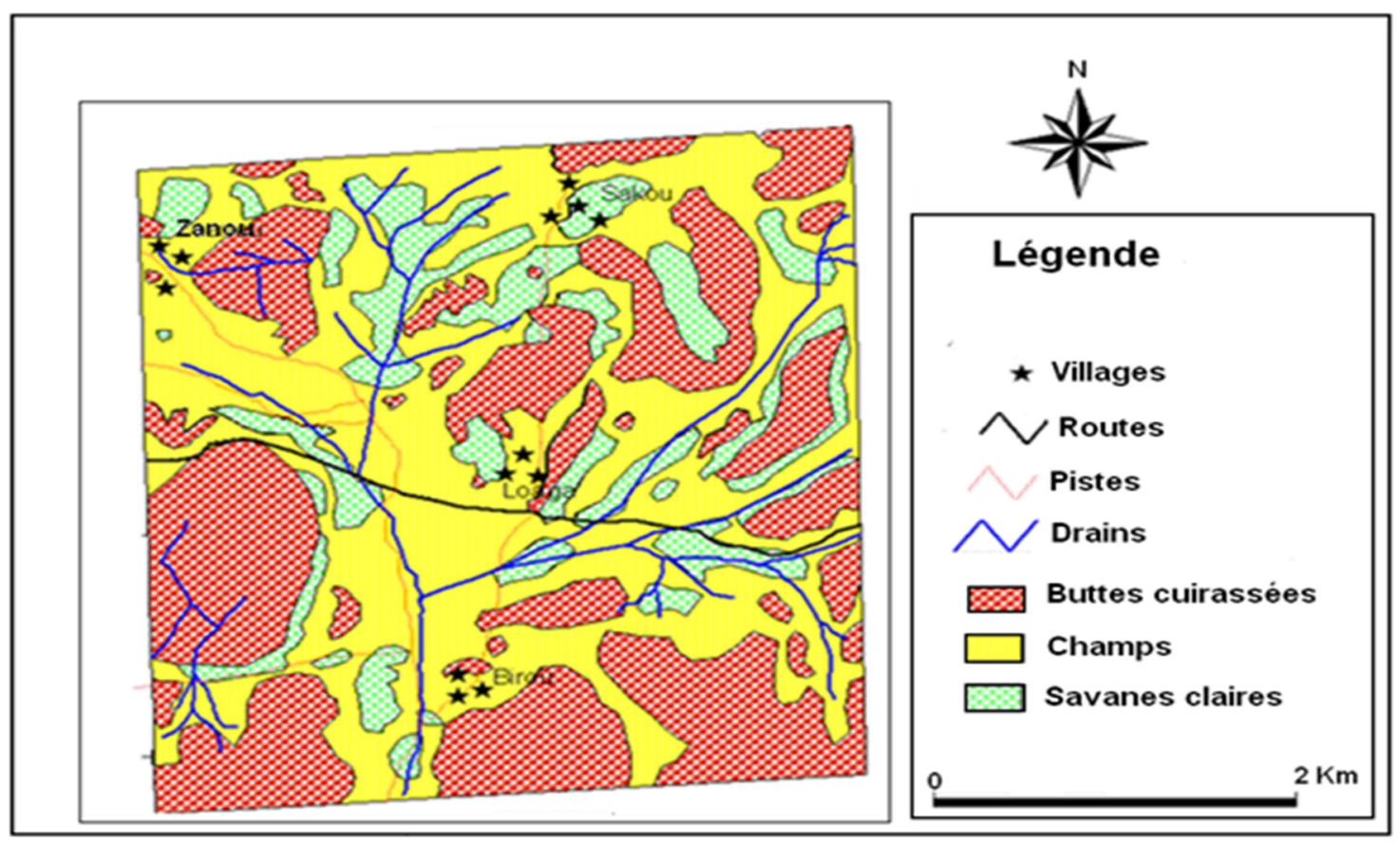

Figure 4: Carte d'occupation des terres de Loaga en 2008. 
Tableau 2: Perceptions paysannes des changements climatiques à Loaga.

\begin{tabular}{lcc}
\hline Variables & Effectifs & Pourcentages (\%) \\
\hline Végétation dense avant 1970 & 41 & 82 \\
Régression de la végétation après 1970 & 47 & 94 \\
Reconstitution de la végétation après 2000 & 37 & 74 \\
Absence de sol nu avant 1970 & 33 & 66 \\
Apparition des sols nus après les sécheresses de 1970 & 43 & 86 \\
Adoption des techniques CES après 1990 & 41 & 82 \\
Sécheresses de 1974-1975 ; 1984-1985 ; 2011 & 31 & 62 \\
Inondations de 1978 & 43 & 86 \\
\hline
\end{tabular}

\section{DISCUSSION}

L'occupation du milieu a connu une dynamique très remarquable de 1982 à 2008 dans le terroir de Loaga. L'analyse des photographies aériennes a révélé une dégradation du couvert végétal qui s'est traduite par l'apparition de $4,5 \%$ de zones nues notées durant la période allant de 1982 à 1995. Cette dégradation du milieu était imputable, à la fois, à l'extension des superficies cultivées avec l'accroissement de la population, au surpâturage et aux variations climatiques (sécheresse de 1974, 1975 et inondations de 1978) (Mertz et al., 2012; Zerbo et al., 2016). Selon Reij et Thiombiano (2003), Sawadogo et al. (2009), les effets cumulés des sécheresses, la pression démographique et le développement de l'élevage ont entraîné une rapide dégradation des écosystèmes sahéliens. La disparition des zones nues observée de 1995 à 2008, pourrait s'expliquer par les effets produits par les aménagements anti-érosifs (cordons pierreux, zaï, demi-lunes) combinés à l'agroforesterie, dans le terroir. Selon divers auteurs (Bayen et al., 2011 ; Somé et al., 2015), les techniques CES comme le zaï, les cordons pierreux et les demi-lunes ont un impact positif sur la récupération du sol et l'augmentation des rendements agricoles.

De 1982 à 1995, l'augmentation des surfaces cultivées $(14,62 \%)$, était négligeable par rapport à la croissance démographique à cause d'un flux migratoire de la population vers les zones urbaines, les zones du pays plus favorables à l'agriculture et vers la Côte d'Ivoire. La baisse des superficies cultivées notée durant la période de 1995 à 2008 serait liée, d'une part, à l'adoption du zaï amélioré combiné au dispositif anti-érosif avec les cordons pierreux et, d'autre part, à l'utilisation d'amendement organique, notamment le compost, générant de meilleurs rendements (Somé et al., 2015 ; Zerbo et al., 2016, Somda et al., 2017). Ces résultats confirment les travaux de Bayen et al. (2011), montrant que les rendements obtenus au niveau des poquets de zaï amendés en compost étaient 12 fois plus élevés que dans les poquets de zaï seul. De même, la production de sorgho dans les parcelles avec apport de compost a été au moins 3 fois supérieure aux parcelles témoins et 1,5 fois supérieure aux traitements avec fumier et ceux avec urée.

La couverture végétale est le meilleur repère pour apprécier les changements du milieu entre différentes périodes dans une région (Belemviré et al., 2008). En effet, de 1982 à 1995, le taux de régression de la savane arbustive a été estimé en moyenne à $37,28 \mathrm{ha} / \mathrm{an}$. Les enquêtes ont révélé à $86 \%$, que les actions anthropiques conjuguées aux aléas climatiques, étaient les principales causes de cette régression de la végétation 
dans le Bam. D'après Mainguet (2003) et Diallo et al. (2017), la dynamique des écosystèmes est étroitement liée aux perturbations climatiques et anthropiques. L'une des conséquences de cette régression de la couverture végétale a été la perte de nombreuses espèces ligneuses utilitaires comme Vitellaria paradoxa, Adansonia digitata, Bombax costatum, Anogeissus leocarpus, Pterocarpus lucens, et Cassia siberiana et les épineux. Par ailleurs, l'expansion des herbacées indicatrices d'une baisse de la fertilité, fréquemment rencontrées dans les champs, confirment la dégradation des sols cultivés (Thombiano et al., 2012 ; Yé et al., 2016).

De 1995 à 2008, l'extension des savanes claires a été en moyenne, de 15,7 ha/an, en raison des jachères pratiquées, mais aussi, du fait des reboisements et des mises en défens opérés qui ont permis de réhabiliter une partie de la savane. Ces résultats confirment les travaux de Zombré (2003) et de Yaméogo et al. (2009) qui soulignent l'efficacité des cordons pierreux ainsi que celle du zaï et du paillage qui seraient favorables à une réinstallation rapide et importante de la végétation. De même, les travaux de Kiema et Sanon (2006) ont montré que les mesures de conservation des eaux et des sols permettaient une amélioration significative du couvert végétal. D'après Roose et al. (1995), Bambara (2011), le zaï constitue une technique qui peut réduire les effets des aléas climatiques, et par conséquent la variabilité interannuelle de la production, tout en augmentant les rendements agricoles et la reprise de la végétation.

\section{Conclusion}

L'étude a clairement montré que les multiples sécheresses, conjuguées à l'explosion démographique ont conduit à une dégradation des ressources naturelles du terroir de Loaga. Les populations ont une perception claire de la dégradation des ressources naturelles, qui s'est accentuée après les sécheresses des années 1970. La tendance à la dégradation du couvert végétal s'est poursuivie entre 1982 et 1995 , et une reconstitution des îlots de végétation à partir des années 1995 et 2008. Les sols ainsi récupérés ont été colonisés par des espèces végétales augmentant la disponibilité fourragère et aussi la biodiversité dans le terroir.

En plus des initiatives endogènes de reconstitution du couvert végétal, une synergie d'action entre les différentes disciplines de gestion des ressources naturelles permettrait de lutter efficacement contre le phénomène de la dégradation. Aussi, un accompagnement des producteurs pour l'acquisition et l'utilisation des intrants agricoles, et des formations sur les techniques $\mathrm{CES} / \mathrm{AGF}$, s'avèrent nécessaires à étendre sur l'ensemble du bassin du fleuve Nakanbé.

\section{CONFLIT D'INTERETS}

Les auteurs déclarent qu'il n'existe pas de conflit d'intérêts sur cet article.

\section{CONTRIBUTIONS DES AUTEURS}

$\mathrm{DM}$ et AAN ont réalisé l'étude, participé aux travaux de terrain et au traitement des données. BK et NPZ ont pris part à la conception du projet de recherche et ont supervisé le travail. Tous ces auteurs ont contribué à la rédaction du manuscrit.

\section{REFERENCES}

Bambara A. 2011. Evaluation de la productivité céréalière des terres aménagées en demi-lunes, diguettes antiérosives et zaï dans le village de Tougou au nord du Burkina Faso. Mémoire du Diplôme d'Etudes Approfondies (DEA), Université de Ouagadougou, $64 \mathrm{p}$.

Bayen P, Traoré S, Bognounou F, Kaiser D, Thiombiano A. 2011. Effet du zaï amélioré sur la productivité du sorgho en zone sahélienne. VertigO - La Revue Electronique en Sciences de l'Environnement, 3(11). http://vertigo. revues.org/11497; Doi : 10.4000/vertigo.11497. 
Bene A, Fournier A. 2014. Végétation naturelle et occupation des terres au Burkina Faso (Afrique de l'ouest). Cinq décennies de changement dans un terroir du pays š̀mè. In Regards scientifiques croisés sur le changement global et le développement - $\quad$ Langue, environnement, culture : Actes du Colloque international de Ouagadougou (8-10 mars 2012). Fabre G, Fournier A, Sanogo L (eds). Sciencesconf.org; 143164.. $<$ hal-00939898 $>$.

Belemviré A, Maïga A, Sawadogo H, Sawadogo M, Ouédraogo S. 2008. Evaluation des impacts biophysiques et socio-économiques des investissements dans les actions de gestion des ressources naturelles au nord du plateau central du Burkina Faso. Rapport de synthèse, étude Sahel, Ouagadougou, 94 p.

Bikienga IM. 2014. Rapport de capitalisation du Projet PANA-BKF-PNUD/FEM Renforcement des capacités pour l'adaptation de la vulnérabilité aux changements climatiques. $44 \mathrm{p}$. http://extwprlegs1.fao.org/docs/pdf/BKF 161723.pdf.

Bilgo A. 2006. Statut organo-minéral et biologique des sols dans les systèmes cultures- jachères naturelles de courtes durées ou améliorée à Andropogon gayanus. Thèse de Doctorat de troisième cycle, Université de Ouagadougou, 177 p.

Borell T. 2000. Etude de la dégradation du couvert végétal dans le sous-bassin versant de Tougo, bassin supérieur de Nakambé, Centre-Nord du BURKINA. Rapport de stage, ETSHER.

Conedera M, Bomio-Pacciorini N, BomioPacciorini P, Sciacca S, Grandi L, Boureima A, Vettraino AM. 2010. Reconstitution des écosystèmes dégradés saheliens. Bois et Forêts des Tropiques, 304(2): 61-71. DOI : bft.cirad.fr/cd/BFT_304_61-71.pdf.

CPCS. 1967. Classification des Sols. Publ. ENSA-GRIGNON: France ; 96 p.
Da DEC, Yacouba H, Yonkeu S. 2008. Unités morphopédologiques et gestion de la fertilité des sols dans le Centre-Nord du Burkina Faso par les populations locales. Int. J. Biol. Chem. Sci., 2(3): 306-315. http://ajol.info/index.php/ijbcs

Diallo S, Nacro HB, N'Diaye A. 2017. Efficience des stratégies endogènes d'adaptation du secteur agricole aux changements climatiques dans le bassin de la région de Thiès (Sénégal). Int. J. Biol. Chem. Sci., 11(2): 707-721. DOI: https://dx.doi.org/10.4314/ijbcs.v11i2.14

Kiema A, Sanon OH. 2006. Restoring the natural pastures in a sahelian region through soil work and sowing of Alysicarpus ovalifolius. Agricultures, 15(5): 41-424. http://cat.inst.fr/?aModele=afficheN

Ibrahim B. 2012. Caractérisation des saisons de pluies au Burkina Faso dans un contexte de changement climatique et évaluation des impacts hydrologiques sur le bassin du Nakanbé. Doctorat, Université Pierre et Marie Curie, 237 p.

Lal R. 2015. Restoring Soil Quality to Mitigate Soil Degradation. Sustainability, $\quad 7$ : 5875-5895. doi:10.3390/su7055875

MAHRH. 2007. Politique nationale de développement durable de l'agriculture irriguée. Rapport principal, Ministère de l'Agriculture de l'Hydraulique et des Ressources Halieutiques (Burkina Faso), $171 \mathrm{p}$.

Mainguet M. 2003. Les Pays Secs. Environnement et Développement. Collection Carrefours: Paris, France; $160 \mathrm{p}$.

Mertz O, D'haen S, Maiga A, Bouzou MI, Barbier B, Diouf A, Diallo D, Da ED, Dabi D. 2012. Climate Variability and Environmental Stress in the Sudan-Sahel Zone of West Africa. Ambio, 41(4): 380392. DOI : 10.1007/s13280-011-0231-8.

Reij C, Thombiano T. 2003. Développement rural et Environnement au Burkina Faso: la Réhabilitation de la Capacité Productive des Terroirs sur la Partie 
Nord du Plateau Central Entre 1980 et 2001. GTZ PATECORE, Ambassade des Pays Bas, USAID ; 82.

Robert E. 2011. Les risques de pertes en terre et en eau dans le bassin versant de la Doubégué (Burkina Faso) pour une gestion intégrée. Thèse de Géographie. Université Michel de Montaigne Bordeaux 3, 533 p. https://hal.archivesouvertes.fr/tel-01090397.

Roose E, Kaboré V, Guenat C. 1995. Le zaï : fonctionnement, limites et amélioration d'une pratique traditionnelle africaine de réhabilitation de la végétation et de la productivité des terres dégradées en région soudano-sahéliennes (Burkina Faso). Cahiers ORSTOM, 158-173.

Sawadogo H, Zombré P, Bock L, Lacroix D. 2009. Evolution de l'occupation du sol de ziga dans le Yatenga (Burkina Faso) à partir de photos aériennes. Télédétection, 8(1): 59-73. https://halshs.archivesouvertes.fr/halshs-00386400

Somda BB, Ouattara B, Serme I, Pouya MB, Lompo F, Taonda SJB, Sédogo PM., 2017. Détermination des doses optimales de fumures organo-minérales en microdose dans la zone soudanosahélienne du Burkina Faso. Int. J. Biol. Chem. Sci., 11(2): 670-683. DOI : https://dx.doi.org/10.4314/ijbcs.v11i2.11

Somé D, Zombré P, Zombré G, Macauley R. 2004. Impact de la technique du zaï sur la production du niébé et sur l'évolution des caractéristiques chimiques des sols très dégradés (Zipéllé) du Burkina Faso. Sécheresse, 15(3): 263-269. http://www.jle.com/fr/revues/sec/e-docs/

Somé D, Hien E, Assigbetse K, Derevon JJ, Masse D. 2015. Dynamique des comportementsdu carbone et de l'azote dans le sol cultivé en niébé et sorgho dans un système de zai en zone Nord soudanienne du Burkina Faso. Int. J. Biol. Chem. Sci., 9(2): 954-969. http://ajol.info/index.php/ijbcs

Thiombiano DNE, Lamien N, Dibong DS, Boussim IJ, Belem B. 2012. Le rôle des espèces ligneuses dans la gestion de la soudure alimentaire au Burkina Faso. Sécheresse, 23(2): 86-93. DOI : $10.1684 / \mathrm{sec} .2012 .0341$

Yaméogo JT, Somé AN, Hien M. 2009. Etude préliminaire à une restauration de sols dégradés en zone soudanienne du Burkina Faso. Sécheresse, 20(4): 397400. DOI : $10.1684 / \mathrm{sec} .2009 .0216$

Yé L, Lata JC, Masse D, Nacro HB, Barot S. 2016. Effets du pâturage sur la biomasse herbacée et sur des paramètres chimiques et biologiques des sols dans une savane arbustive au Burkina Faso. Int. J. Biol. Chem. Sci., 10(6): 2539-2554. DOI: http://dx.doi.org/10.4314/ijbcs.v10i6.11

Zerbo L, Nacro HB, Yao-Kouamé A, Sédogo PM. 2016. Connaissances et perceptions locales de la dynamique des cuirasses ferrugineuses : Etude de cas en zone Nord-soudanienne et Sud-soudanienne du Burkina Faso. Int. J. Biol. Chem. Sci., 10(6): 2754-2767. DOI: http://dx.doi.org/10.4314/ijbcs.v10i6.28

Zombré NP. 2003. Les sols très dégradés "Zipella' du centre nord du Burkina Faso: Dynamique, caractéristiques morpho-bio-pédologique et impacts des technique de restauration. Thèse doctorat d'état ès Sciences Naturelles, Université de Ouagadougou Burkina Faso, 374 p.

Zombré NP. 2006. Evolution de l'occupation des terres et localisation des sols dans le Centre-Nord du Burkina. Télédétection, 5(4): 285-297. 